\title{
Helicobacter pylori Phage Screening
}

Filipa F. Vale*, António P. A. Matos**, Patrícia Carvalho***, and Jorge M. B. Vítor****

* Engineering Faculty, Portuguese Catholic University, Estrada Octávio Pato, 2635-631 Rio de Mouro, Portugal, e-mail: filipavale@fe.ucp.pt;

** Biomaterials Department, Dental Medical School, University of Lisbon and Curry Cabral Hospital, Lisbon, Portugal;

*** Department of Materials Engineering, Technical University of Lisbon, 1049-001 Lisbon, Portugal;

**** CECF (iMed), Pharmacy Faculty University of Lisbon, Av. Prof. Gama Pinto 1649-019 Lisboa, Portugal.

Helicobacter pylori is a helical shaped Gram-negative bacterium that colonizes the human stomach. It is associated with several human pathologies, such as gastritis, peptic ulcer, and gastric cancer. The standard first-line treatment is a one week triple therapy: the association of two antibiotics, most frequently amoxicillin and clarithromycin, and a proton pump inhibitor. Despite the evolution of the treatment strategy, quadruple therapy, there is an increasing percentage of failure of the antibiotic therapy, due to antibiotics resistance [1]. Phage therapy is the therapeutic use of lytic bacteriophages to treat pathogenic bacterial infections and $H$. pylori is a good target [2]. However there are no available phage collections, and $H$. pylori phages description is diminutive on literature [3].

We developed a new $H$. pylori phage screening procedure, as follows: a suspension of $1 \mathrm{~g}$ of human faeces in $9 \mathrm{ml}$ of SSM buffer (sodium chloride $0,1 \mathrm{M}$; calcium chloride $1 \mathrm{mM}$; magnesium sulphate $10 \mathrm{mM}$; Tris- $\mathrm{HCl} \mathrm{pH} 7,550 \mathrm{mM}$; gelatine $0,01 \%$ ) was incubated at room temperature with $150 \mathrm{rpm}$ agitation, after vortex for 1 minute. After centrifugation at $4000 \mathrm{rpm}, 4^{\circ} \mathrm{C}$ during 5 minutes (Allegra 6R, Beckman-Coulter, rotor GA6), the supernatant was recovered and filtered through a PES $0.22 \mathrm{~m}$ sterile filter (SLGP033RS, Millex GP, Millipore, USA). Finally the filtered supernatant was concentrated using PES Vivaspin 20 pore 100.000 MWCO (Sartorius AG, Goettingen, Germany). Seven different faeces concentrates were tested with 201 of each H. pylori strain suspension in BHI (Oxoid, UK) during 15 minutes at room temperature. The negative control was inoculated with 51 of SSM buffer. The suspensions were inoculated in Hp agar selective medium (Biogerm, Porto, Portugal) and incubated at $37^{\circ} \mathrm{C}$ in an anaerobic jar (Oxoid or BBL) with a gas generator system (CampyGen, Oxoid) [4]. The Petri dishes were observed every 48 hours during 10 days. Lytic plaques (Fig.1) were observed in 4 H. pylori strains: BAPOUI, JP1/95, 1603/05, and Mex228. UV irradiation of positive strains induced the production of lytic plates on strain JP1/95, which suggest the presence of a lysogenic phage (Fig.5).

Cultures presenting lytic plaques were analysed by Scanning Electron Microscopy (JEOL 7001F SEM) (Fig.2) and Transmission Electron Microscopy (JEM 100SXTEM) (Figs. 3-5). Cell cultures growing over agar were fixed by cutting a region of interest containing both the agar and the overlaying cell culture, and immersing the agar layer in glutaraldehyde fixative in a covered Petri dish, without disturbing the delicate culture layer. After overnight fixation, the level of the fixative was slowly raised above the top surface of the agar allowing the fixed cell culture layer to detach from the agar and to float over the liquid fixative, where it was left for 1 hour for additional fixation. The floating cell culture forms a resistant membrane that can be easily collected with a glass coverslip and pre-embedded in $2 \%$ agar for further processing. Further fixation, embedding and 
sectioning were performed according to standard transmission electron microscopy procedures. Negative staining was performed in samples adsorbed to formvar or formvar-carbon grids and stained with $1 \%$ aqueous uranyl acetate. Our results disclosed the presence of virus-like particles by negative staining in the infectious samples. TEM images show bacteriophage-like structures with a diameter approximately $100 \mathrm{~nm}$ without tail (Fig. 3 and 4) from strain BAPOUI inoculated with a human faeces concentrate, and 15nm filaments (Fig 5) obtained from JP1/95 UV irradiated. Both might represent phage particles. Further studies are under way to obtain additional structural and molecular evidence for the viral nature of the observed particles.

\section{References}

[1] J. G. Kusters et al. Clin.Microbiol. Rev. 19 (2006) 449.

[2] S. Matsuzaki et al. J. Infect Chemother. 11 (2005) 211.

[3] H. E. Heintschel et al. J. Med. Microbiol. 38 (1993) 245.

[4] F. F. Vale and J. M. B. Vítor. Appl. Environ. Microbiol. 73 (2007) 4243.

[5] This work was partially funded by New England Biolabs, USA
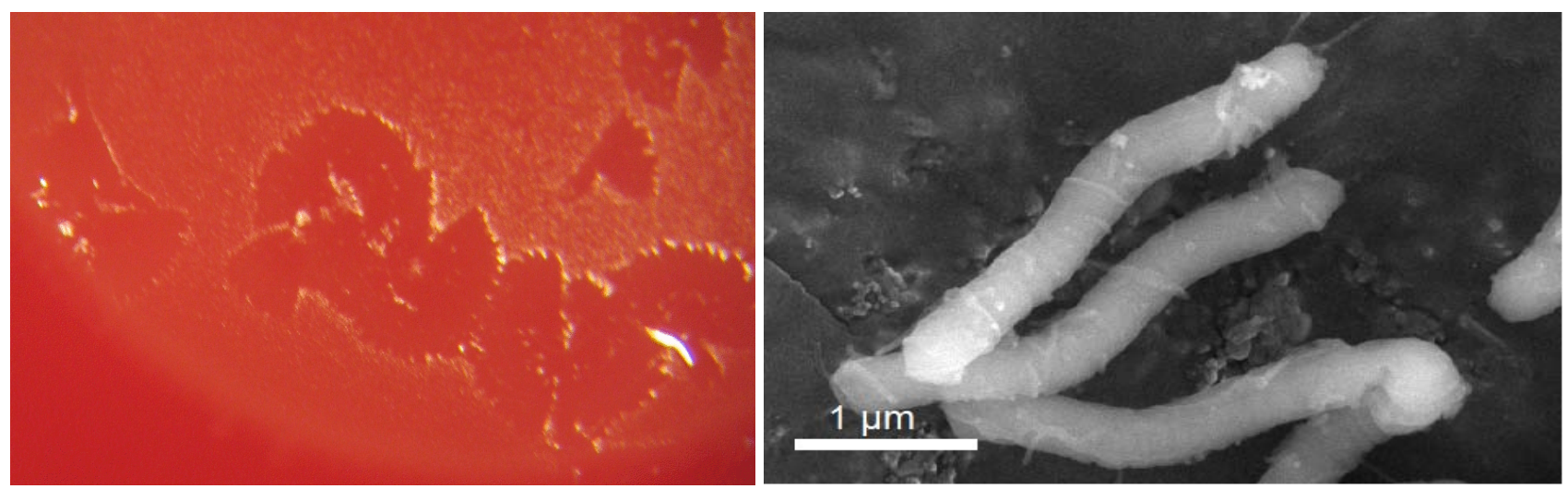

Fig. 1 - Phage plaques in H. pylori culture.

Fig. 2 - H. pylori cultured cells cells seen by SEM.

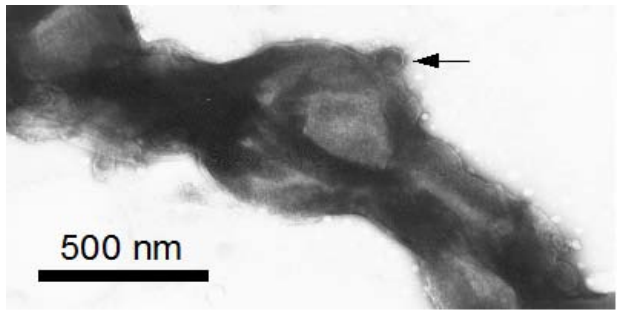

Fig. 3 - Negative staining of $H$. pylori cell showing a polyhedral virus-like particle (arrow).
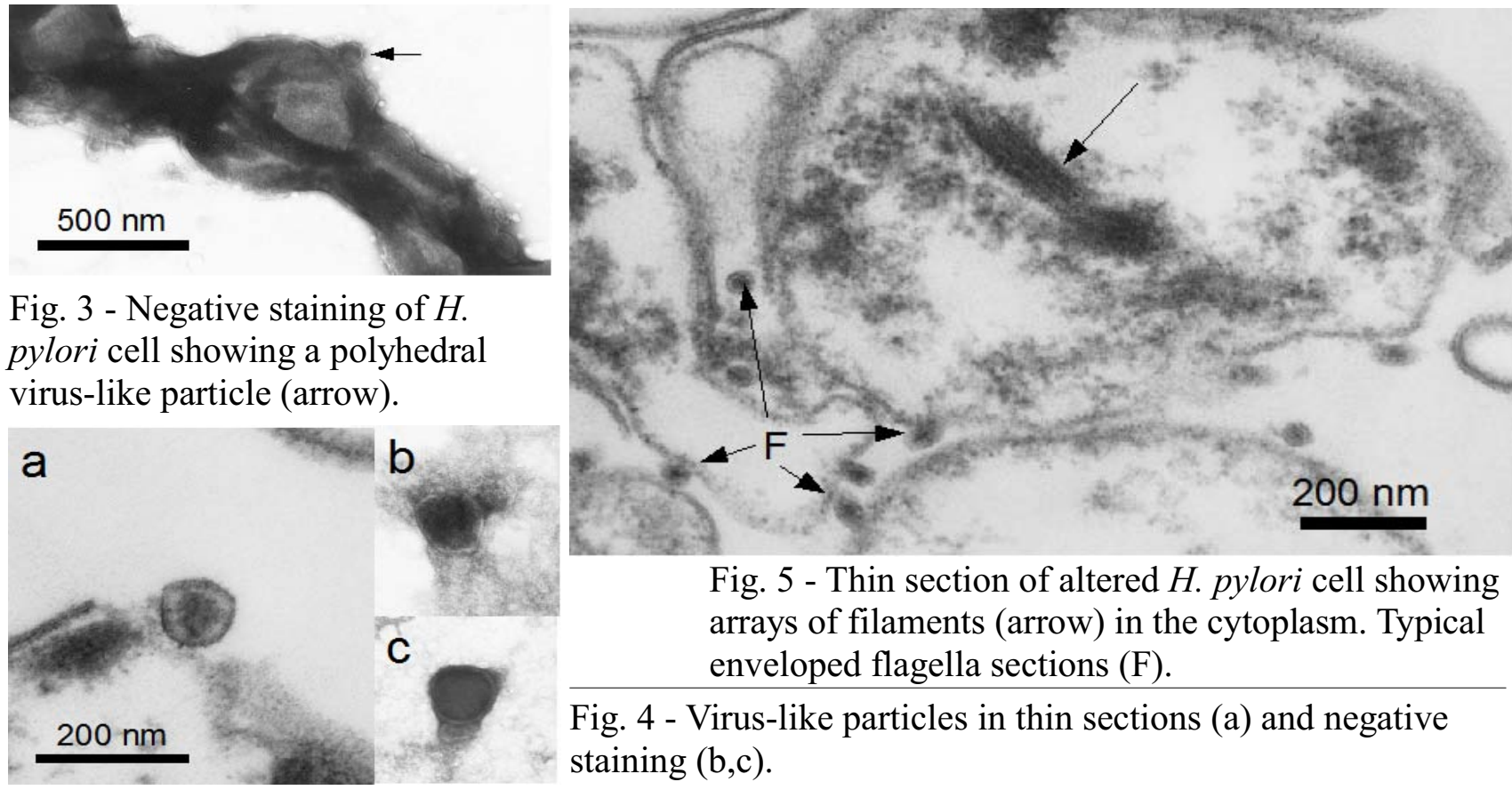

Fig. 5 - Thin section of altered H. pylori cell showing arrays of filaments (arrow) in the cytoplasm. Typical enveloped flagella sections (F).

Fig. 4 - Virus-like particles in thin sections (a) and negative staining $(b, c)$. 